\title{
Migrant Boat Mini Challenge Award: Simple and Effective Integrated Display Geo-Temporal Analysis of Migrant Boats
}

\author{
R. Miklin, T. Lipić* \\ Dept. of Telecom.-FER \\ Univ. of Zagreb, Croatia
}

\author{
Z. Konyha ${ }^{\dagger}$ \\ VRVis Research \\ Vienna, Austria
}

\author{
M. Berić \\ Dept. of Telecom.-FER \\ Univ. of Zagreb, Croatia
}

\author{
W. Freiler, K. Matković ${ }^{\S}$ \\ VRVis Research \\ Vienna, Austria
}

\author{
D. Gračanin ${ }^{\text {II }}$ \\ Virginia Tech, USA
}

\begin{abstract}
We provide a description of the tools and techniques used in our analysis of the VAST 2008 Challenge dealing with mass movement of persons departing Isla Del Sueño on boats for the United States during 2005-2007. We used visual analytics to explore migration patterns, characterize the choice and evolution of landing sites, characterize the geographical patterns of interdictions and determine the successful landing rate. Our ComVis tool, in connection with some helper applications and Google Earth, allowed us to explore geo-temporal characteristics of the data set and answer the challenge questions. The ComVis project file captures the visual analysis context and facilitates better collaboration among team members.
\end{abstract}

Keywords: Visual analytics, geo-temporal data.

Index Terms: I.3.0 [Computer Graphics]: General-; I.3.6 [Computer Graphics]: Methodology and Techniques-(Interaction techniques); J.4.1 [Social and Behavioral Sciences]: Sociology-

\section{INTRODUCTION}

Visual analysis of geo-temporal data is a navigation in an unfamiliar space of geographic metadata and a semantic reasoning to support the seeker in the definition of the search criteria [2]. We describe the use of ComVis, an interactive visualization tool providing multiple, linked views and composite brushing. ComVis has a number of standard views (histograms, scatter plots and parallel coordinates) while time series data can be displayed in function graph views [3]. It also provides composite brushes (in the same or in different views) constructed using sequences of AND, OR and SUB operations. This dynamic filtering is a key feature for interactive analysis [1]. We also developed a C\# application to parse the input Migrand Data.xml, pre-process data for use in ComVis and to generate a . kml file to show encounters using Google Earth.

\section{Data Set Characteristics}

The data set, the migrant boat records, includes the interdiction records collected by the United States Coast Guard and information from other sources about illegal landings on shore. Each record has several fields, including $(x, y)$ coordinates, date and type (interdiction or landing). We categorized the data in several ways in order to gain better insight.

\footnotetext{
*e-mail: r.miklin@gmail.com, tomislav.lipic@fer.hr

†e-mail: konyha@vrvis.at

†e-mail: marko.beric@fer.hr

§e-mail: freiler@vrvis.at, matkovic@vrvis.at

Ile-mail: gracanin@vt.edu
}

First, we analyzed separately successful landings and interdictions to get an insight where are the actual landing sites and where the migrants are trying to go. Second, we studied encounter time data at different time scales (individual encounter, month, quarter, year) to determine possible trends. and temporal distribution of landing sites. Third, we used encounter coordinates data to determine a geographical distribution of landing sites. Finally, we checked launch coordinates and vessel types but that section of the data set had very limited impact on the overall analysis results.

\section{Analysis Procedures}

Due to the geo-temporal nature of the data set, we used Google Earth to augment the views provided by ComVis (Figure 1). That allows us to provide the real-world context for the data set and make it easier to identify geographical distribution.

\subsection{Geo-Temporal Distribution}

Since ComVis provides multiple linked views, we used it to quickly specify (using a composite brush) data categories of interest. Figure 1a) illustrates the use of this feature to provide a geo-temporal view of the landing sites. Four linked views were used. The first view (upper left), a histogram of record types, is used for brushing (selecting the data records of interest - landing sites). Since each record contains coordinates ( $x$ and $y$ ), we use a 2D plot view to indicate geographical distribution (lower right view). The selected data records are shown in red while the other records are shown in grey.

The remaining two views provide temporal distributions using histograms. The first one (upper right view) provides a finer resolution (quarterly) that indicates seasonal variations in number of landing sites - majority are during spring and summer months. The second one (lower left view) provides a coarser resolution (yearly) that indicates the overall trend - a significant increase in number of landing over the period of three years.

When the landing sites coordinates were viewed in Google Earth we determined that there are two countries affected, United States (Florida) and Mexico (islands Contoy and Mujeres).

\subsection{Composite Brushing}

Composite brushing allows us to combine several search criteria (brushes) to find answer for more specific questions. While Figure 1 provides an overall geographical distribution, we would like to find out how that distribution depends on the date of the events. Figure 2 shows the landings that occurred in 2005 . We first brushed the landing sites (upper left view) and then year 2005 (lower left view). The remaining two views now show that the landings took place in south Florida (lower right view) and that the number of landings has been increasing constantly (upper right view). We can similarly determine that in Year 2 the landings spread to west Florida and Mexico while in Year 3 most of the landings were in Mexico. 


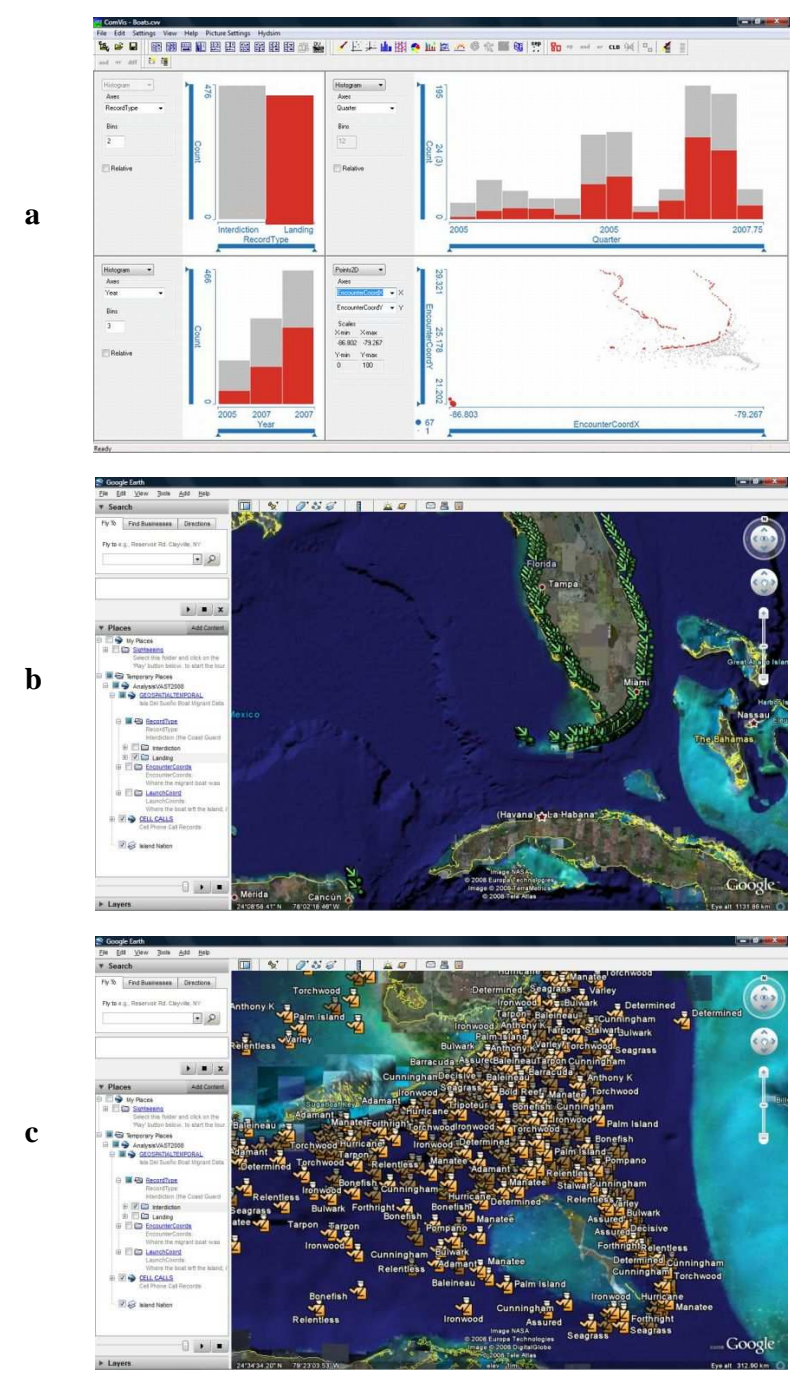

Figure 1: a) ComVis multiple linked view of the data set. We brush the landing records (upper left view), provide temporal distribution at two scales, by quarters (upper right view) and by years (lower left view) and provide "geographical" distribution (lower right view) for the landing sites records (red points) while still indicating other records (grey points). b) Google Earth view of the landing sites providing an enhanced view of the geographical distribution (red points in a). c) Google Earth view of the non-landing sites (interdiction locations) providing an enhanced view of the geographical distribution (grey points in a)

\subsection{Table View}

In some situations we need the raw data records resulting from a (composite) brush. A raw data view (table view) allows sorting and filtering based on the individual field values (Figure 3).

\subsection{Collaboration}

ComVis composite brush feature, combined with information stored in ComVis project file, facilitates better collaboration among team mebers. In other words, ComVis lends itself well to off-line collaboration. While it is (still) a single-user application, it has ability to capture the state of the visual analysis session (brushes, views, etc.) and store it (together with the data being analyzed) in a single project file (. CVv file). Such file provides the collaborators the common context and framework for visual analysis.

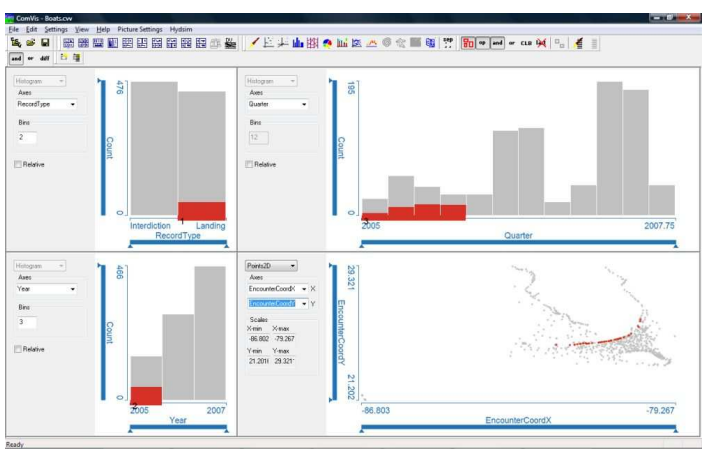

Figure 2: The original brush of the landing records from Figure 1a (upper left view) is now combined with another brush, year 2005 records (lower left view), to provide a specific "geographical" distribution (lower right view) and temporal distribution in four quarters of 2005 (upper right view).

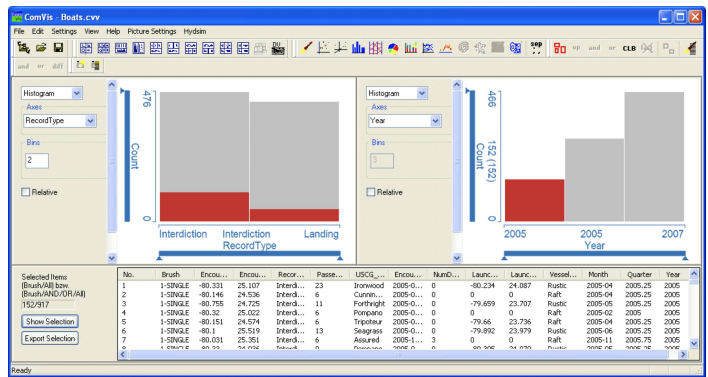

Figure 3: Brushing year 2005 records (upper right view) immediately provides a distribution between interdictions and landings (upper left view). The corresponding table view (lower view) allows direct sorting based on the individual field values.

For example, a geographical distribution view (Figure 1a) created by one collaborator can be used by other collaborator as a starting point to create a temporal distribution view in Figure 2 by refining the initial brush (selection) while the third collaborator is focusing on the year 2005 data records (Figure 3). ComVis made it possible for our team members located in Vienna (Austria), Zagreb (Croatia), and Blacksburg (USA) to better leverage regular communication channels (audio/video conferences, workshops) using the common visual analysis context captured in . Cvv files.

\section{Conclusion}

We used ComVis, an interactive visualization tool with multiple linked views and composite brushing, to perform visual analysis of geo-temporal data. The geographical distribution view was complemented by the corresponding Google Earth view to provide realworld context of the analyzed data set.

\section{REFERENCES}

[1] C. Ahlberg and B. Shneiderman. Visual information seeking: tight coupling of dynamic query filters with starfield displays. In $\mathrm{CHI}$ '94: Conference companion on Human factors in computing systems, pages 313-321, New York, NY, USA, 1994. ACM Press.

[2] R. Albertoni, A. Bertone, and M. D. Martino. Visualization and semantic analysis of geographic metadata. In GIR '05: Proceedings of the 2005 workshop on Geographic information retrieval, pages 9-16, New York, NY, USA, Nov. 4 2005. ACM Press.

[3] Z. Konyha, K. Matković, D. Gračanin, M. Jelović, and H. Hauser. Interactive visual analysis of families of function graphs. IEEE Transaction on Visualization and Computer Graphics, 12(6):1373-1385, 2006. 